\title{
Stability of the 7-3 Compressor Circuit for Wallace Tree. Part $\mathbf{I}^{\mathbb{1}}$
}

\author{
Katsumi Wasaki \\ Shinshu University \\ Nagano, Japan
}

\begin{abstract}
Summary. To evaluate our formal verification method on a real-size calculation circuit, in this article, we continue to formalize the concept of the 7-3 Compressor (STC) Circuit [6] for Wallace Tree [11, to define the structures of calculation units for a very fast multiplication algorithm for VLSI implementation 10. We define the circuit structure of the tree constructions of the Generalized Full Adder Circuits (GFAs). We then successfully prove its circuit stability of the calculation outputs after four and six steps. The motivation for this research is to establish a technique based on formalized mathematics and its applications for calculation circuits with high reliability, and to implement the applications of the reliable logic synthesizer and hardware compiler $[5$.
\end{abstract}

MSC: 68M07 68W35 68V20

Keywords: arithmetic processor; high order compressor; high-speed multiplier; Wallace tree; logic circuit stability

MML identifier: WALLACE1, version: 8.1.09 5.60.1371

\section{INTRODUCTION}

Since calculation models of the arithmetic logic unit based on many sorted algebra have been proposed, we continue to verify the structure and design of these circuits using the Mizar [2, 3], 44 proof checking system. Actually, the stability of circuit primitives is proved based on the definitions and theorems on logic operations, hardware gates, and signal lines [8], 9], [7].

\footnotetext{
${ }^{1}$ This work has been partially supported by the JSPS KAKENHI Grant Number 19K11821, Japan. 
The various concepts for the Boolean operations, the logic gate elements needed to define the digital circuit, and the connections are defined and have been proved [1]. For logic gate elements that compose a calculation circuit using many Boolean operations, we have prepared a practical collection of logic gates [13]. To construct the adder circuit structure for the RSD numeric representation, we then formalized the definitions and properties of the Generalized Full Adder Circuits (GFAs) to have three inputs and two outputs [14]. Since we have to scale the size of evaluation up to this formal verification method on a real-size calculation circuit, we have already completed formalize the concept of the 4-2 Binary Addition Cell primitives (FTAs) [12] to construct the structures of calculation units for a very fast multiplication algorithm for VLSI implementation [10.

There is the Wallace tree multiplication method [11] as achieved high-speed multiplier, which is connected like the tree using the usual full adder (FA) circuit cell. Since it transforms the Wallace tree multiplication method to improve the high-speed computation and circuit regularity, there is also a refinement multiplication method using the 7-3 Compressor Circuit [6].

We show the component symbol and the block diagram of a 7-3 Compressor Circuit implementation in Figure 1 and Figure 2 using four GFAs. First two GFAs take six of the seven inputs $(\mathrm{x} 1, \mathrm{x} 2, \mathrm{x} 3, \mathrm{x} 5, \mathrm{x} 6, \mathrm{x} 7)$ and generate two sum $(\mathrm{A} 1, \mathrm{~A} 2)$ and two carry outputs $(\mathrm{C} 1, \mathrm{C} 2)$ in Layer-I (after 2-steps). The sum outputs are combined with the seventh input $(\mathrm{x} 4)$ in another GFA to generate the s0 output of the 7-3 Compressor in Layer-II (after 4-steps). The carry output of this GFA is combined with the carry outputs from the two first level GFAs using fourth GFA to yield s1 and s2, with weights of two and four respectively in Layer-III (after 6-steps).

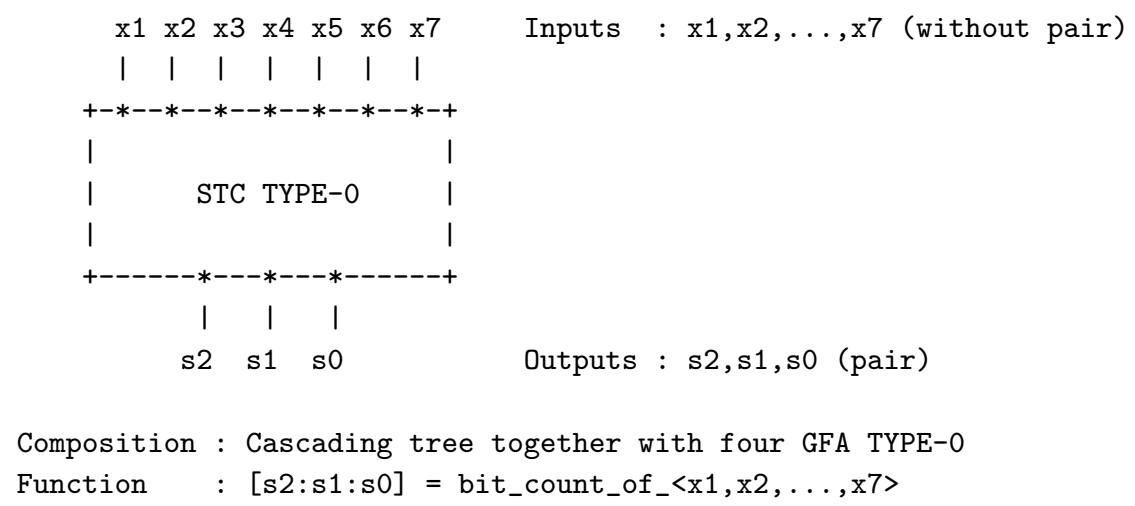

Fig.1 7-3 Compressor Circuit (Seven-to-Three Compressor:STC): TYPE-0, Component Symbol. 


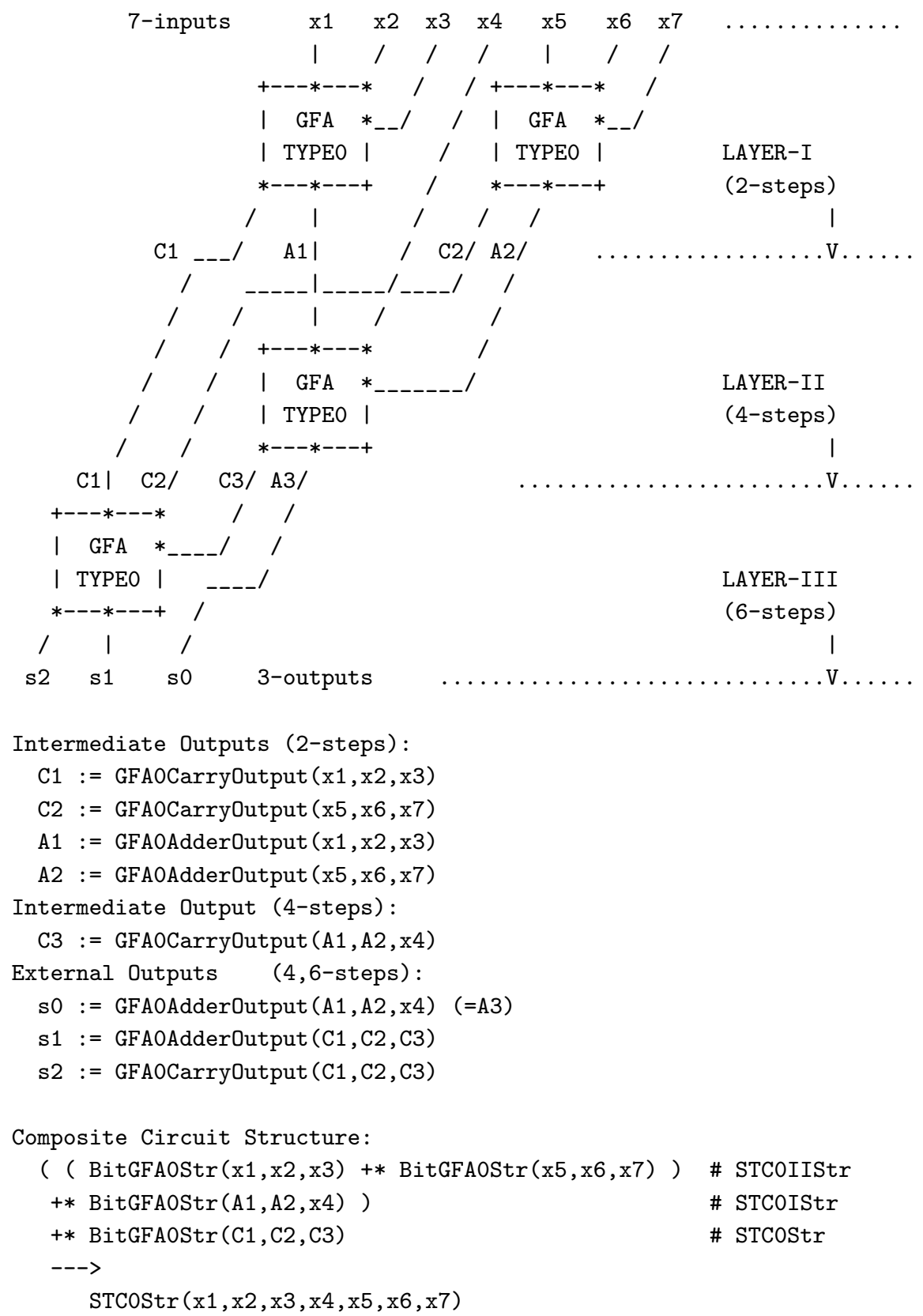

Fig.2 7-3 Compressor Circuit, Block Diagram and Calculation Stability: Following(s,6) is stable. 


\section{Properties of 'Intermediate' STC Circuit Structure (LAYER-I)}

Let $x_{1}, x_{2}, x_{3}, x_{4}$ be non pair objects. Let us note that $\left\{x_{1}, x_{2}, x_{3}, x_{4}\right\}$ has no pairs.

Let $x_{5}$ be a non pair object. Observe that $\left\{x_{1}, x_{2}, x_{3}, x_{4}, x_{5}\right\}$ has no pairs.

Let $x_{6}$ be a non pair object. Let us note that $\left\{x_{1}, x_{2}, x_{3}, x_{4}, x_{5}, x_{6}\right\}$ has no pairs.

Let $x_{7}$ be a non pair object. One can verify that $\left\{x_{1}, x_{2}, x_{3}, x_{4}, x_{5}, x_{6}, x_{7}\right\}$ has no pairs.

Let $x_{1}, x_{2}, x_{3}, x_{5}, x_{6}, x_{7}$ be sets. The functor $\operatorname{STC} 0 \operatorname{IIStr}\left(x_{1}, x_{2}, x_{3}, x_{5}, x_{6}, x_{7}\right)$ yielding an unsplit, non void, strict, non empty many sorted signature with arity held in gates and Boolean denotation held in gates is defined by the term

(Def. 1) $\operatorname{BitGFA0Str}\left(x_{1}, x_{2}, x_{3}\right)+\cdot \operatorname{BitGFA0Str}\left(x_{5}, x_{6}, x_{7}\right)$.

The functor STC0IICirc $\left(x_{1}, x_{2}, x_{3}, x_{5}, x_{6}, x_{7}\right)$ yielding a strict, Boolean circuit of $\operatorname{STC} 0 \operatorname{IIStr}\left(x_{1}, x_{2}, x_{3}, x_{5}, x_{6}, x_{7}\right)$ with denotation held in gates is defined by the term

(Def. 2) $\operatorname{BitGFA0Circ}\left(x_{1}, x_{2}, x_{3}\right)+\cdot \operatorname{BitGFA0Circ}\left(x_{5}, x_{6}, x_{7}\right)$.

Let us consider sets $x_{1}, x_{2}, x_{3}, x_{5}, x_{6}, x_{7}$. Now we state the propositions:

(1) InnerVertices(STC0IIStr $\left.\left(x_{1}, x_{2}, x_{3}, x_{5}, x_{6}, x_{7}\right)\right)=\left(\left(\left\{\left\langle\left\langle x_{1}, x_{2}\right\rangle\right.\right.\right.\right.$, xor $\left._{2}\right\rangle$, GFA0AdderOutput $\left.\left(x_{1}, x_{2}, x_{3}\right)\right\} \cup\left\{\left\langle\left\langle x_{1}, x_{2}\right\rangle\right.\right.$, and $\left.{ }_{2}\right\rangle,\left\langle\left\langle x_{2}, x_{3}\right\rangle\right.$, and $\left.{ }_{2}\right\rangle,\left\langle\left\langle x_{3}\right.\right.$, $\left.x_{1}\right\rangle$, and $\left.{ }_{2}\right\rangle$, GFA0CarryOutput $\left.\left.\left(x_{1}, x_{2}, x_{3}\right)\right\}\right) \cup\left\{\left\langle\left\langle x_{5}, x_{6}\right\rangle\right.\right.$, xor $\left.{ }_{2}\right\rangle$, GFA0Ad$\left.\left.\operatorname{derOutput}\left(x_{5}, x_{6}, x_{7}\right)\right\}\right) \cup\left\{\left\langle\left\langle x_{5}, x_{6}\right\rangle, \operatorname{and}_{2}\right\rangle,\left\langle\left\langle x_{6}, x_{7}\right\rangle\right.\right.$, $\left.\operatorname{and}_{2}\right\rangle,\left\langle\left\langle x_{7}, x_{5}\right\rangle\right.$, $\left.\operatorname{and}_{2}\right\rangle$, GFA0CarryOutput $\left.\left(x_{5}, x_{6}, x_{7}\right)\right\}$.

(2) InnerVertices( $\left.\operatorname{STC} 0 \operatorname{IIStr}\left(x_{1}, x_{2}, x_{3}, x_{5}, x_{6}, x_{7}\right)\right)$ is a binary relation.

Let us consider non pair sets $x_{1}, x_{2}, x_{3}, x_{5}, x_{6}, x_{7}$. Now we state the propositions:

(3) InputVertices(STC0IIStr $\left.\left(x_{1}, x_{2}, x_{3}, x_{5}, x_{6}, x_{7}\right)\right)=\left\{x_{1}, x_{2}, x_{3}, x_{5}, x_{6}, x_{7}\right\}$.

(4) InputVertices(STC0IIStr $\left.\left(x_{1}, x_{2}, x_{3}, x_{5}, x_{6}, x_{7}\right)\right)$ has no pairs.

Let us consider sets $x_{1}, x_{2}, x_{3}, x_{5}, x_{6}, x_{7}$. Now we state the propositions:

(5) $x_{1}, x_{2}, x_{3}, x_{5}, x_{6}, x_{7},\left\langle\left\langle x_{1}, x_{2}\right\rangle\right.$, xor $\left.x_{2}\right\rangle$, GFA0AdderOutput $\left(x_{1}, x_{2}, x_{3}\right)$, $\left\langle\left\langle x_{1}, x_{2}\right\rangle, \operatorname{and}_{2}\right\rangle,\left\langle\left\langle x_{2}, x_{3}\right\rangle, \operatorname{and}_{2}\right\rangle,\left\langle\left\langle x_{3}, x_{1}\right\rangle, \operatorname{and}_{2}\right\rangle, \operatorname{GFA} 0 \operatorname{CarryOutput}\left(x_{1}\right.$, $\left.x_{2}, x_{3}\right),\left\langle\left\langle x_{5}, x_{6}\right\rangle\right.$, xor $\left._{2}\right\rangle$, GFA0AdderOutput $\left(x_{5}, x_{6}, x_{7}\right),\left\langle\left\langle x_{5}, x_{6}\right\rangle, \operatorname{and}_{2}\right\rangle$, $\left\langle\left\langle x_{6}, x_{7}\right\rangle, \operatorname{and}_{2}\right\rangle,\left\langle\left\langle x_{7}, x_{5}\right\rangle, \operatorname{and}_{2}\right\rangle$, GFA0CarryOutput $\left(x_{5}, x_{6}, x_{7}\right) \in$ the carrier of $\operatorname{STC} 0 \operatorname{IIStr}\left(x_{1}, x_{2}, x_{3}, x_{5}, x_{6}, x_{7}\right)$.

(6) $\left\langle\left\langle x_{1}, x_{2}\right\rangle, \operatorname{xor}_{2}\right\rangle$, GFA0AdderOutput $\left(x_{1}, x_{2}, x_{3}\right),\left\langle\left\langle x_{1}, x_{2}\right\rangle, \operatorname{and}_{2}\right\rangle,\left\langle\left\langle x_{2}\right.\right.$, $\left.\left.x_{3}\right\rangle, \operatorname{and}_{2}\right\rangle,\left\langle\left\langle x_{3}, x_{1}\right\rangle, \operatorname{and}_{2}\right\rangle$, GFA0CarryOutput $\left(x_{1}, x_{2}, x_{3}\right),\left\langle\left\langle x_{5}, x_{6}\right\rangle\right.$, xor $\left._{2}\right\rangle$, 
GFA0AdderOutput $\left(x_{5}, x_{6}, x_{7}\right),\left\langle\left\langle x_{5}, x_{6}\right\rangle, \operatorname{and}_{2}\right\rangle,\left\langle\left\langle x_{6}, x_{7}\right\rangle, \operatorname{and}_{2}\right\rangle,\left\langle\left\langle x_{7}, x_{5}\right\rangle\right.$, $\left.\operatorname{and}_{2}\right\rangle$, GFA0CarryOutput $\left(x_{5}, x_{6}, x_{7}\right) \in \operatorname{InnerVertices}\left(\operatorname{STC} 0 \operatorname{IIStr}\left(x_{1}, x_{2}\right.\right.$, $\left.x_{3}, x_{5}, x_{6}, x_{7}\right)$ ). The theorem is a consequence of (1).

(7) Let us consider non pair sets $x_{1}, x_{2}, x_{3}, x_{5}, x_{6}, x_{7}$. Then $x_{1}, x_{2}, x_{3}, x_{5}$, $x_{6}, x_{7} \in \operatorname{InputVertices}\left(\operatorname{STC} 0 \operatorname{IIStr}\left(x_{1}, x_{2}, x_{3}, x_{5}, x_{6}, x_{7}\right)\right)$. The theorem is a consequence of (3).

Let $x_{1}, x_{2}, x_{3}, x_{5}, x_{6}, x_{7}$ be sets. The functors: STC0IICarryOutC1 $\left(x_{1}, x_{2}, x_{3}\right.$, $\left.x_{5}, x_{6}, x_{7}\right)$, STC0IIAdderOutA1 $\left(x_{1}, x_{2}, x_{3}, x_{5}, x_{6}, x_{7}\right)$, STC0IICarryOutC2 $\left(x_{1}\right.$, $\left.x_{2}, x_{3}, x_{5}, x_{6}, x_{7}\right)$, and STC0IIAdderOutA2 $\left(x_{1}, x_{2}, x_{3}, x_{5}, x_{6}, x_{7}\right)$ yielding elements of InnerVertices(STC0IIStr $\left.\left(x_{1}, x_{2}, x_{3}, x_{5}, x_{6}, x_{7}\right)\right)$ are defined by terms

(Def. 3) GFA0CarryOutput $\left(x_{1}, x_{2}, x_{3}\right)$,

(Def. 4) GFA0AdderOutput $\left(x_{1}, x_{2}, x_{3}\right)$,

(Def. 5) GFA0CarryOutput $\left(x_{5}, x_{6}, x_{7}\right)$,

(Def. 6) GFA0AdderOutput $\left(x_{5}, x_{6}, x_{7}\right)$,

respectively. Now we state the propositions:

(8) Let us consider non pair sets $x_{1}, x_{2}, x_{3}, x_{5}, x_{6}, x_{7}$, a state $s$ of STC0IICirc $\left(x_{1}, x_{2}, x_{3}, x_{5}, x_{6}, x_{7}\right)$, and elements $a_{1}, a_{2}, a_{3}, a_{5}, a_{6}, a_{7}$ of Boolean. Suppose $a_{1}=s\left(x_{1}\right)$ and $a_{2}=s\left(x_{2}\right)$ and $a_{3}=s\left(x_{3}\right)$ and $a_{5}=s\left(x_{5}\right)$ and $a_{6}=s\left(x_{6}\right)$ and $a_{7}=s\left(x_{7}\right)$. Then

(i) $($ Following $(s, 2))\left(\mathrm{STC0IICarryOutC1}\left(x_{1}, x_{2}, x_{3}, x_{5}, x_{6}, x_{7}\right)\right)=\left(a_{1} \wedge\right.$ $\left.a_{2} \vee a_{2} \wedge a_{3}\right) \vee a_{3} \wedge a_{1}$, and

(ii) $($ Following $(s, 2))\left(\operatorname{STC0IIAdderOutA1}\left(x_{1}, x_{2}, x_{3}, x_{5}, x_{6}, x_{7}\right)\right)=\left(a_{1} \oplus\right.$ $\left.a_{2}\right) \oplus a_{3}$, and

(iii) $($ Following $(s, 2))\left(\mathrm{STC} 0 \mathrm{IICarryOutC} 2\left(x_{1}, x_{2}, x_{3}, x_{5}, x_{6}, x_{7}\right)\right)=\left(a_{5} \wedge\right.$ $\left.a_{6} \vee a_{6} \wedge a_{7}\right) \vee a_{7} \wedge a_{5}$, and

(iv) $($ Following $(s, 2))\left(\operatorname{STC0IIAdderOutA2}\left(x_{1}, x_{2}, x_{3}, x_{5}, x_{6}, x_{7}\right)\right)=\left(a_{5} \oplus\right.$ $\left.a_{6}\right) \oplus a_{7}$, and

(v) $($ Following $(s, 2))\left(x_{1}\right)=a_{1}$, and

(vi) $($ Following $(s, 2))\left(x_{2}\right)=a_{2}$, and

(vii) $(\operatorname{Following}(s, 2))\left(x_{3}\right)=a_{3}$, and

(viii) (Following $(s, 2))\left(x_{5}\right)=a_{5}$, and

(ix) $($ Following $(s, 2))\left(x_{6}\right)=a_{6}$, and

$(\mathrm{x})(\operatorname{Following}(s, 2))\left(x_{7}\right)=a_{7}$.

The theorem is a consequence of (7).

(9) Let us consider non pair sets $x_{1}, x_{2}, x_{3}, x_{5}, x_{6}, x_{7}$, and a state $s$ of $\operatorname{STC0IICirc}\left(x_{1}, x_{2}, x_{3}, x_{5}, x_{6}, x_{7}\right)$. Then Following $(s, 2)$ is stable. 


\section{Properties of 'Intermediate' STC Circuit Structure (LAYER-II)}

Let $x_{1}, x_{2}, x_{3}, x_{4}, x_{5}, x_{6}, x_{7}$ be sets. The functor $\operatorname{STC} 0 \operatorname{IStr}\left(x_{1}, x_{2}, x_{3}, x_{4}, x_{5}\right.$, $\left.x_{6}, x_{7}\right)$ yielding an unsplit, non void, strict, non empty many sorted signature with arity held in gates and Boolean denotation held in gates is defined by the term

(Def. 7) STC0IIStr $\left(x_{1}, x_{2}, x_{3}, x_{5}, x_{6}, x_{7}\right)+\cdot \operatorname{BitGFA0Str}\left(\right.$ GFA0AdderOutput $\left(x_{1}\right.$, $\left.x_{2}, x_{3}\right)$, GFA0AdderOutput $\left.\left(x_{5}, x_{6}, x_{7}\right), x_{4}\right)$.

The functor STC0ICirc $\left(x_{1}, x_{2}, x_{3}, x_{4}, x_{5}, x_{6}, x_{7}\right)$ yielding a strict, Boolean circuit of $\operatorname{STC} 0 \operatorname{IStr}\left(x_{1}, x_{2}, x_{3}, x_{4}, x_{5}, x_{6}, x_{7}\right)$ with denotation held in gates is defined by the term

(Def. 8) STC0IICirc $\left(x_{1}, x_{2}, x_{3}, x_{5}, x_{6}, x_{7}\right)+\cdot \operatorname{BitGFA0Circ(GFA0AdderOutput}\left(x_{1}\right.$, $\left.x_{2}, x_{3}\right)$, GFA0AdderOutput $\left.\left(x_{5}, x_{6}, x_{7}\right), x_{4}\right)$.

Let us consider sets $x_{1}, x_{2}, x_{3}, x_{4}, x_{5}, x_{6}, x_{7}$.

Now we state the propositions:

(10) InnerVertices(STC0IStr $\left.\left(x_{1}, x_{2}, x_{3}, x_{4}, x_{5}, x_{6}, x_{7}\right)\right)=$ $\left\{\left\langle\left\langle x_{1}, x_{2}\right\rangle\right.\right.$, xor $\left._{2}\right\rangle$, GFA0AdderOutput $\left.\left(x_{1}, x_{2}, x_{3}\right)\right\} \cup$ $\left\{\left\langle\left\langle x_{1}, x_{2}\right\rangle, \operatorname{and}_{2}\right\rangle,\left\langle\left\langle x_{2}, x_{3}\right\rangle, \operatorname{and}_{2}\right\rangle,\left\langle\left\langle x_{3}, x_{1}\right\rangle, \operatorname{and}_{2}\right\rangle\right.$, GFA0CarryOutput $\left(x_{1}\right.$, $\left.\left.x_{2}, x_{3}\right)\right\} \cup$ $\left\{\left\langle\left\langle x_{5}, x_{6}\right\rangle\right.\right.$, xor $\left._{2}\right\rangle$, GFA0AdderOutput $\left.\left(x_{5}, x_{6}, x_{7}\right)\right\} \cup$ $\left\{\left\langle\left\langle x_{5}, x_{6}\right\rangle, \operatorname{and}_{2}\right\rangle,\left\langle\left\langle x_{6}, x_{7}\right\rangle, \operatorname{and}_{2}\right\rangle,\left\langle\left\langle x_{7}, x_{5}\right\rangle, \operatorname{and}_{2}\right\rangle\right.$, GFA0CarryOutput $\left(x_{5}\right.$, $\left.\left.x_{6}, x_{7}\right)\right\} \cup$ $\left\{\left\langle\left\langle\right.\right.\right.$ GFA0AdderOutput $\left(x_{1}, x_{2}, x_{3}\right)$, GFA0AdderOutput $\left.\left.\left(x_{5}, x_{6}, x_{7}\right)\right\rangle, \operatorname{xor}_{2}\right\rangle$, GFA0AdderOutput(GFA0AdderOutput $\left(x_{1}, x_{2}, x_{3}\right)$, GFA0AdderOutput $\left.\left.\left(x_{5}, x_{6}, x_{7}\right), x_{4}\right)\right\} \cup$ $\left\{\left\langle\left\langle\right.\right.\right.$ GFA0AdderOutput $\left(x_{1}, x_{2}, x_{3}\right)$, GFA0AdderOutput $\left.\left.\left(x_{5}, x_{6}, x_{7}\right)\right\rangle, \operatorname{and}_{2}\right\rangle$, $\left\langle\left\langle\right.\right.$ GFA0AdderOutput $\left.\left(x_{5}, x_{6}, x_{7}\right), x_{4}\right\rangle$, and $\left._{2}\right\rangle,\left\langle\left\langle x_{4}\right.\right.$, GFA0AdderOutput $\left.\left(x_{1}, x_{2}, x_{3}\right)\right\rangle$, and $\left.{ }_{2}\right\rangle$, GFA0CarryOutput(GFA0AdderOutput $\left(x_{1}, x_{2}, x_{3}\right)$, GFA0AdderOutput $\left.\left.\left(x_{5}, x_{6}, x_{7}\right), x_{4}\right)\right\}$.

The theorem is a consequence of (1).

(11) InnerVertices(STC0IStr $\left(x_{1}, x_{2}, x_{3}, x_{4}, x_{5}, x_{6}, x_{7}\right)$ ) is a binary relation.

(12) Let us consider non pair sets $x_{1}, x_{2}, x_{3}, x_{5}, x_{6}, x_{7}$, and a set $x_{4}$. Suppose $x_{4} \neq\left\langle\left\langle\operatorname{GFA0AdderOutput}\left(x_{1}, x_{2}, x_{3}\right)\right.\right.$, GFA0AdderOutput $\left.\left(x_{5}, x_{6}, x_{7}\right)\right\rangle$, xor $\left._{2}\right\rangle$ and $x_{4} \neq\left\langle\left\langle\right.\right.$ GFA0AdderOutput $\left(x_{1}, x_{2}, x_{3}\right)$, GFA0AdderOutput $\left(x_{5}\right.$, $\left.\left.x_{6}, x_{7}\right)\right\rangle$, $\left.\operatorname{and}_{2}\right\rangle$ and $x_{4} \notin \operatorname{InnerVertices}\left(\operatorname{STC} 0 \operatorname{ISStr}\left(x_{1}, x_{2}, x_{3}, x_{5}, x_{6}, x_{7}\right)\right)$. Then InputVertices(STC0IStr $\left.\left(x_{1}, x_{2}, x_{3}, x_{4}, x_{5}, x_{6}, x_{7}\right)\right)=\left\{x_{1}, x_{2}, x_{3}, x_{4}\right.$, $\left.x_{5}, x_{6}, x_{7}\right\}$. The theorem is a consequence of (1) and (3).

Let us consider non pair sets $x_{1}, x_{2}, x_{3}, x_{4}, x_{5}, x_{6}, x_{7}$. 
(13) InputVertices(STC0IStr $\left.\left(x_{1}, x_{2}, x_{3}, x_{4}, x_{5}, x_{6}, x_{7}\right)\right)=\left\{x_{1}, x_{2}, x_{3}, x_{4}, x_{5}, x_{6}\right.$, $\left.x_{7}\right\}$. The theorem is a consequence of (12).

(14) InputVertices(STC0IStr $\left.\left(x_{1}, x_{2}, x_{3}, x_{4}, x_{5}, x_{6}, x_{7}\right)\right)$ has no pairs. The theorem is a consequence of (13).

Let us consider sets $x_{1}, x_{2}, x_{3}, x_{4}, x_{5}, x_{6}, x_{7}$.

(15) $x_{1}, x_{2}, x_{3}, x_{4}, x_{5}, x_{6}, x_{7},\left\langle\left\langle x_{1}, x_{2}\right\rangle, \operatorname{xor}_{2}\right\rangle$, GFA0AdderOutput $\left(x_{1}, x_{2}, x_{3}\right)$, $\left\langle\left\langle x_{1}, x_{2}\right\rangle, \operatorname{and}_{2}\right\rangle,\left\langle\left\langle x_{2}, x_{3}\right\rangle, \operatorname{and}_{2}\right\rangle,\left\langle\left\langle x_{3}, x_{1}\right\rangle, \operatorname{and}_{2}\right\rangle, \operatorname{GFA0CarryOutput}\left(x_{1}\right.$, $\left.x_{2}, x_{3}\right)$, 〈 $\left\langle\right.$ GFA0AdderOutput $\left(x_{1}, x_{2}, x_{3}\right)$, GFA0AdderOutput $\left.\left(x_{5}, x_{6}, x_{7}\right)\right\rangle$, $\left.\operatorname{xor}_{2}\right\rangle$, GFA0AdderOutput(GFA0AdderOutput $\left(x_{1}, x_{2}, x_{3}\right)$, GFA0Adder Output $\left.\left(x_{5}, x_{6}, x_{7}\right), x_{4}\right)$, 《/GFA0AdderOutput $\left(x_{1}, x_{2}, x_{3}\right)$, GFA0AdderOut$\left.\left.\operatorname{put}\left(x_{5}, x_{6}, x_{7}\right)\right\rangle, \operatorname{and}_{2}\right\rangle,\left\langle\left\langle\mathrm{GFA0AdderOutput}\left(x_{5}, x_{6}, x_{7}\right), x_{4}\right\rangle, \operatorname{and}_{2}\right\rangle,\left\langle\left\langle x_{4}\right.\right.$, GFA0AdderOutput $\left.\left(x_{1}, x_{2}, x_{3}\right)\right\rangle$, and $\left.\operatorname{an}_{2}\right\rangle \in$ the carrier of $\operatorname{STC0IStr}\left(x_{1}, x_{2}\right.$, $\left.x_{3}, x_{4}, x_{5}, x_{6}, x_{7}\right)$.

And also GFA0CarryOutput(GFA0AdderOutput $\left(x_{1}, x_{2}, x_{3}\right)$, GFA0 AdderOutput $\left.\left(x_{5}, x_{6}, x_{7}\right), x_{4}\right),\left\langle\left\langle x_{5}, x_{6}\right\rangle, \operatorname{xor}_{2}\right\rangle, \operatorname{GFA0AdderOutput}\left(x_{5}, x_{6}\right.$, $\left.x_{7}\right),\left\langle\left\langle x_{5}, x_{6}\right\rangle\right.$, and $\left._{2}\right\rangle,\left\langle\left\langle x_{6}, x_{7}\right\rangle\right.$, and $\left._{2}\right\rangle,\left\langle\left\langle x_{7}, x_{5}\right\rangle, \operatorname{and}_{2}\right\rangle$, GFA0CarryOut$\operatorname{put}\left(x_{5}, x_{6}, x_{7}\right) \in$ the carrier of $\operatorname{STC} 0 \operatorname{IStr}\left(x_{1}, x_{2}, x_{3}, x_{4}, x_{5}, x_{6}, x_{7}\right)$.

The theorem is a consequence of (5).

(16) $\left\langle\left\langle x_{1}, x_{2}\right\rangle, \operatorname{xor}_{2}\right\rangle$, GFA0AdderOutput $\left(x_{1}, x_{2}, x_{3}\right),\left\langle\left\langle x_{1}, x_{2}\right\rangle, \operatorname{and}_{2}\right\rangle,\left\langle\left\langle x_{2}\right.\right.$, $\left.x_{3}\right\rangle$, and and $\left._{2}\right\rangle\left\langle x_{3}, x_{1}\right\rangle$, and 2$\rangle$, GFA0CarryOutput $\left(x_{1}, x_{2}, x_{3}\right)$, 〈GFA0Adder Output $\left(x_{1}, x_{2}, x_{3}\right)$, GFA0AdderOutput $\left.\left(x_{5}, x_{6}, x_{7}\right)\right\rangle$, xor 2$\rangle$, GFA0Adder Output(GFA0AdderOutput $\left(x_{1}, x_{2}, x_{3}\right)$, GFA0AdderOutput $\left.\left(x_{5}, x_{6}, x_{7}\right), x_{4}\right)$, 《 GFA0AdderOutput $\left(x_{1}, x_{2}, x_{3}\right)$, GFA0AdderOutput $\left.\left(x_{5}, x_{6}, x_{7}\right)\right\rangle$, and $\left.\operatorname{an}_{2}\right\rangle$, $\left\langle\left\langle\right.\right.$ GFA0AdderOutput $\left.\left.\left(x_{5}, x_{6}, x_{7}\right), x_{4}\right\rangle, \operatorname{and}_{2}\right\rangle,\left\langle\left\langle x_{4}\right.\right.$, GFA0AdderOutput $\left.\left(x_{1}, x_{2}, x_{3}\right)\right\rangle$, and $\left.{ }_{2}\right\rangle$, GFA0CarryOutput(GFA0AdderOutput $\left(x_{1}, x_{2}, x_{3}\right)$, GFA0AdderOutput $\left.\left(x_{5}, x_{6}, x_{7}\right), x_{4}\right),\left\langle\left\langle x_{5}, x_{6}\right\rangle\right.$, xor 2$\rangle$, GFA0AdderOutput $\left(x_{5}, x_{6}, x_{7}\right),\left\langle\left\langle x_{5}, x_{6}\right\rangle, \operatorname{and}_{2}\right\rangle,\left\langle\left\langle x_{6}, x_{7}\right\rangle, \operatorname{and}_{2}\right\rangle,\left\langle\left\langle x_{7}, x_{5}\right\rangle, \operatorname{and}_{2}\right\rangle$, GFA0Carry $\operatorname{Output}\left(x_{5}, x_{6}, x_{7}\right) \in \operatorname{InnerVertices}\left(\operatorname{STC} 0 \operatorname{IStr}\left(x_{1}, x_{2}, x_{3}, x_{4}, x_{5}, x_{6}, x_{7}\right)\right)$. The theorem is a consequence of (10).

(17) Let us consider non pair sets $x_{1}, x_{2}, x_{3}, x_{5}, x_{6}, x_{7}$, and a set $x_{4}$. Suppose $x_{4} \neq\left\langle\left\langle\right.\right.$ GFA0AdderOutput $\left(x_{1}, x_{2}, x_{3}\right)$, GFA0AdderOutput $\left.\left(x_{5}, x_{6}, x_{7}\right)\right\rangle$, xor $\left._{2}\right\rangle$ and $x_{4} \neq\left\langle\left\langle\right.\right.$ GFA0AdderOutput $\left(x_{1}, x_{2}, x_{3}\right), \operatorname{GFA0AdderOutput}\left(x_{5}\right.$,

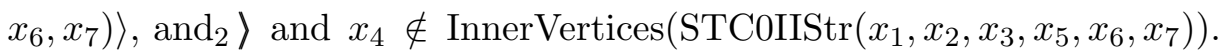
Then $x_{1}, x_{2}, x_{3}, x_{4}, x_{5}, x_{6}, x_{7} \in \operatorname{InputVertices}\left(\operatorname{STC} 0 \operatorname{IStr}\left(x_{1}, x_{2}, x_{3}, x_{4}, x_{5}\right.\right.$, $\left.x_{6}, x_{7}\right)$ ). The theorem is a consequence of (12).

(18) Let us consider non pair sets $x_{1}, x_{2}, x_{3}, x_{4}, x_{5}, x_{6}, x_{7}$. Then $x_{1}, x_{2}$, $x_{3}, x_{4}, x_{5}, x_{6}, x_{7} \in \operatorname{InputVertices}\left(\operatorname{STC} 0 \operatorname{IStr}\left(x_{1}, x_{2}, x_{3}, x_{4}, x_{5}, x_{6}, x_{7}\right)\right)$. The theorem is a consequence of (13). 
Let $x_{1}, x_{2}, x_{3}, x_{4}, x_{5}, x_{6}, x_{7}$ be sets. The functors: STC0ICarryOutC1 $\left(x_{1}, x_{2}\right.$, $\left.x_{3}, x_{4}, x_{5}, x_{6}, x_{7}\right)$, STC0ICarryOutC2 $\left(x_{1}, x_{2}, x_{3}, x_{4}, x_{5}, x_{6}, x_{7}\right)$, STC0ICarry

OutC3 $\left(x_{1}, x_{2}, x_{3}, x_{4}, x_{5}, x_{6}, x_{7}\right)$, and STC0IAdderOutA3 $\left(x_{1}, x_{2}, x_{3}, x_{4}, x_{5}, x_{6}\right.$,

$\left.x_{7}\right)$ yielding elements of InnerVertices(STC0IStr $\left.\left(x_{1}, x_{2}, x_{3}, x_{4}, x_{5}, x_{6}, x_{7}\right)\right)$ are defined by terms

(Def. 9) GFA0CarryOutput $\left(x_{1}, x_{2}, x_{3}\right)$,

(Def. 10) GFA0CarryOutput $\left(x_{5}, x_{6}, x_{7}\right)$,

(Def. 11) GFA0CarryOutput(GFA0AdderOutput $\left(x_{1}, x_{2}, x_{3}\right)$, GFA0AdderOutput $\left.\left(x_{5}, x_{6}, x_{7}\right), x_{4}\right)$,

(Def. 12) GFA0AdderOutput(GFA0AdderOutput $\left(x_{1}, x_{2}, x_{3}\right)$, GFA0AdderOutput $\left.\left(x_{5}, x_{6}, x_{7}\right), x_{4}\right)$,

respectively.

Now we state the propositions:

(19) Let us consider non pair sets $x_{1}, x_{2}, x_{3}, x_{4}, x_{5}, x_{6}, x_{7}$, a state $s$ of $\operatorname{STC0ICirc}\left(x_{1}, x_{2}, x_{3}, x_{4}, x_{5}, x_{6}, x_{7}\right)$, and elements $a_{1}, a_{2}, a_{3}, a_{4}, a_{5}, a_{6}$, $a_{7}$ of Boolean. Suppose $a_{1}=s\left(x_{1}\right)$ and $a_{2}=s\left(x_{2}\right)$ and $a_{3}=s\left(x_{3}\right)$ and $a_{4}=s\left(x_{4}\right)$ and $a_{5}=s\left(x_{5}\right)$ and $a_{6}=s\left(x_{6}\right)$ and $a_{7}=s\left(x_{7}\right)$. Then

(i) $($ Following $(s, 2))\left(\operatorname{STC0ICarryOutC1}\left(x_{1}, x_{2}, x_{3}, x_{4}, x_{5}, x_{6}, x_{7}\right)\right)=\left(a_{1} \wedge\right.$ $\left.a_{2} \vee a_{2} \wedge a_{3}\right) \vee a_{3} \wedge a_{1}$, and

(ii) $($ Following $(s, 2))\left(\mathrm{STC0ICarryOutC2}\left(x_{1}, x_{2}, x_{3}, x_{4}, x_{5}, x_{6}, x_{7}\right)\right)=\left(a_{5} \wedge\right.$ $\left.a_{6} \vee a_{6} \wedge a_{7}\right) \vee a_{7} \wedge a_{5}$, and

(iii) $($ Following $(s, 4))\left(\mathrm{STC0ICarryOutC} 3\left(x_{1}, x_{2}, x_{3}, x_{4}, x_{5}, x_{6}, x_{7}\right)\right)=$ $\left(\left(\left(a_{1} \oplus a_{2}\right) \oplus a_{3}\right) \wedge\left(\left(a_{5} \oplus a_{6}\right) \oplus a_{7}\right) \vee\left(\left(a_{5} \oplus a_{6}\right) \oplus a_{7}\right) \wedge a_{4}\right) \vee a_{4} \wedge\left(\left(a_{1} \oplus\right.\right.$ $\left.\left.a_{2}\right) \oplus a_{3}\right)$, and

(iv) $($ Following $(s, 4))\left(\operatorname{STC0IAdderOutA3}\left(x_{1}, x_{2}, x_{3}, x_{4}, x_{5}, x_{6}, x_{7}\right)\right)=$ $\left.\left(\left(\left(\left(a_{1} \oplus a_{2}\right) \oplus a_{3}\right) \oplus a_{4}\right) \oplus a_{5}\right) \oplus a_{6}\right) \oplus a_{7}$, and

(v) $($ Following $(s, 4))\left(x_{1}\right)=a_{1}$, and

(vi) (Following $(s, 4))\left(x_{2}\right)=a_{2}$, and

(vii) $($ Following $(s, 4))\left(x_{3}\right)=a_{3}$, and

(viii) (Following $(s, 4))\left(x_{4}\right)=a_{4}$, and

(ix) $($ Following $(s, 4))\left(x_{5}\right)=a_{5}$, and

(x) $($ Following $(s, 4))\left(x_{6}\right)=a_{6}$, and

(xi) (Following $(s, 4))\left(x_{7}\right)=a_{7}$.

(20) Let us consider non pair sets $x_{1}, x_{2}, x_{3}, x_{4}, x_{5}, x_{6}, x_{7}$, and a state $s$ of $\operatorname{STC0ICirc}\left(x_{1}, x_{2}, x_{3}, x_{4}, x_{5}, x_{6}, x_{7}\right)$. Then Following $(s, 4)$ is stable. The theorem is a consequence of (9). 


\section{Properties of STC Circuit Structure (LAYER-III)}

Let $x_{1}, x_{2}, x_{3}, x_{4}, x_{5}, x_{6}, x_{7}$ be sets. The functor $\operatorname{STC} \operatorname{CStr}\left(x_{1}, x_{2}, x_{3}, x_{4}, x_{5}\right.$, $\left.x_{6}, x_{7}\right)$ yielding an unsplit, non void, strict, non empty many sorted signature with arity held in gates and Boolean denotation held in gates is defined by the term

(Def. 13) STC0IStr $\left(x_{1}, x_{2}, x_{3}, x_{4}, x_{5}, x_{6}, x_{7}\right)+\cdot \operatorname{BitGFA0Str}\left(\mathrm{STC} 0 I C a r r y O u t C 1\left(x_{1}\right.\right.$, $\left.x_{2}, x_{3}, x_{4}, x_{5}, x_{6}, x_{7}\right)$, STC0ICarryOutC2 $\left(x_{1}, x_{2}, x_{3}, x_{4}, x_{5}, x_{6}, x_{7}\right)$, STC0I CarryOutC3 $\left.\left(x_{1}, x_{2}, x_{3}, x_{4}, x_{5}, x_{6}, x_{7}\right)\right)$.

The functor STC0Circ $\left(x_{1}, x_{2}, x_{3}, x_{4}, x_{5}, x_{6}, x_{7}\right)$ yielding a strict, Boolean circuit of $\operatorname{STC} 0 \operatorname{Str}\left(x_{1}, x_{2}, x_{3}, x_{4}, x_{5}, x_{6}, x_{7}\right)$ with denotation held in gates is defined by the term

(Def. 14) STC0ICirc $\left(x_{1}, x_{2}, x_{3}, x_{4}, x_{5}, x_{6}, x_{7}\right)+$ - BitGFA0Circ(STC0ICarryOutC1 $\left(x_{1}, x_{2}, x_{3}, x_{4}, x_{5}, x_{6}, x_{7}\right)$, STC0ICarryOutC2 $\left(x_{1}, x_{2}, x_{3}, x_{4}, x_{5}, x_{6}, x_{7}\right)$, STC0ICarryOutC3 $\left.\left(x_{1}, x_{2}, x_{3}, x_{4}, x_{5}, x_{6}, x_{7}\right)\right)$.

Let us consider sets $x_{1}, x_{2}, x_{3}, x_{4}, x_{5}, x_{6}, x_{7}$. Now we state the propositions:

(21) InnerVertices $\left(\operatorname{STC} 0 \operatorname{Str}\left(x_{1}, x_{2}, x_{3}, x_{4}, x_{5}, x_{6}, x_{7}\right)\right)=$ InnerVertices(STC0IStr $\left.\left(x_{1}, x_{2}, x_{3}, x_{4}, x_{5}, x_{6}, x_{7}\right)\right) \cup$ $\left\{\left\langle\left\langle\right.\right.\right.$ STC0ICarryOutC1 $\left(x_{1}, x_{2}, x_{3}, x_{4}, x_{5}, x_{6}, x_{7}\right)$, STC0ICarryOutC2 $\left(x_{1}, x_{2}\right.$, $\left.\left.x_{3}, x_{4}, x_{5}, x_{6}, x_{7}\right)\right\rangle$, xor $\left._{2}\right\rangle$, GFA0AdderOutput(STC0ICarryOutC1 $\left(x_{1}, x_{2}\right.$, $\left.x_{3}, x_{4}, x_{5}, x_{6}, x_{7}\right)$, STC0ICarryOutC2 $\left(x_{1}, x_{2}, x_{3}, x_{4}, x_{5}, x_{6}, x_{7}\right), \mathrm{STC} 0 \mathrm{ICa}-$ $\left.\left.\operatorname{rryOutC} 3\left(x_{1}, x_{2}, x_{3}, x_{4}, x_{5}, x_{6}, x_{7}\right)\right)\right\} \cup$ $\left\{\left\langle\left\langle\right.\right.\right.$ STC0ICarryOutC1 $\left(x_{1}, x_{2}, x_{3}, x_{4}, x_{5}, x_{6}, x_{7}\right)$, STC0ICarryOutC2 $\left(x_{1}, x_{2}\right.$, $\left.\left.\left.x_{3}, x_{4}, x_{5}, x_{6}, x_{7}\right)\right\rangle, \operatorname{and}_{2}\right\rangle,\left\langle\left\langle\mathrm{STC} 0 \mathrm{ICarryOutC} 2\left(x_{1}, x_{2}, x_{3}, x_{4}, x_{5}, x_{6}, x_{7}\right)\right.\right.$, STC0ICarryOutC3 $\left.\left(x_{1}, x_{2}, x_{3}, x_{4}, x_{5}, x_{6}, x_{7}\right)\right\rangle$, and $\left.{ }_{2}\right\rangle$, $\langle\langle$ STC0ICarryOut $\mathrm{C} 3\left(x_{1}, x_{2}, x_{3}, x_{4}, x_{5}, x_{6}, x_{7}\right)$, STC0ICarryOutC1 $\left.\left(x_{1}, x_{2}, x_{3}, x_{4}, x_{5}, x_{6}, x_{7}\right)\right\rangle$, $\left.\operatorname{and}_{2}\right\rangle$, GFA0CarryOutput(STC0ICarryOutC1 $\left(x_{1}, x_{2}, x_{3}, x_{4}, x_{5}, x_{6}, x_{7}\right)$, STC0ICarryOutC2 $\left(x_{1}, x_{2}, x_{3}, x_{4}, x_{5}, x_{6}, x_{7}\right)$, STC0ICarryOutC3 $\left(x_{1}, x_{2}\right.$, $\left.\left.\left.x_{3}, x_{4}, x_{5}, x_{6}, x_{7}\right)\right)\right\}$.

(22) InnerVertices $\left(\operatorname{STC} 0 \operatorname{Str}\left(x_{1}, x_{2}, x_{3}, x_{4}, x_{5}, x_{6}, x_{7}\right)\right)=\left\{\left\langle\left\langle x_{1}, x_{2}\right\rangle\right.\right.$, xor $\left._{2}\right\rangle$, GFA0AdderOutput $\left.\left(x_{1}, x_{2}, x_{3}\right)\right\} \cup\left\{\left\langle\left\langle x_{1}, x_{2}\right\rangle, \operatorname{and}_{2}\right\rangle,\left\langle\left\langle x_{2}, x_{3}\right\rangle\right.\right.$, and $\left.\ln _{2}\right\rangle,\left\langle\left\langle x_{3}\right.\right.$, $\left.x_{1}\right\rangle$, and $\left.{ }_{2}\right\rangle$, GFA0CarryOutput $\left.\left(x_{1}, x_{2}, x_{3}\right)\right\} \cup\left\{\left\langle\left\langle x_{5}, x_{6}\right\rangle\right.\right.$, xor $\left._{2}\right\rangle$, GFA0Adder $\left.\operatorname{Output}\left(x_{5}, x_{6}, x_{7}\right)\right\} \cup\left\{\left\langle\left\langle x_{5}, x_{6}\right\rangle, \operatorname{and}_{2}\right\rangle,\left\langle\left\langle x_{6}, x_{7}\right\rangle, \operatorname{and}_{2}\right\rangle,\left\langle\left\langle x_{7}, x_{5}\right\rangle, \operatorname{and}_{2}\right\rangle\right.$, GFA0CarryOutput $\left.\left(x_{5}, x_{6}, x_{7}\right)\right\} \cup\left\{\left\langle\left\langle\right.\right.\right.$ GFA0AdderOutput $\left(x_{1}, x_{2}, x_{3}\right)$, GFA0 AdderOutput $\left.\left(x_{5}, x_{6}, x_{7}\right)\right\rangle$, xor 2$\rangle$, GFA0AdderOutput(GFA0AdderOutput $\left(x_{1}, x_{2}, x_{3}\right)$, GFA0AdderOutput $\left.\left.\left(x_{5}, x_{6}, x_{7}\right), x_{4}\right)\right\} \cup\{\langle\langle$ GFA0AdderOutput

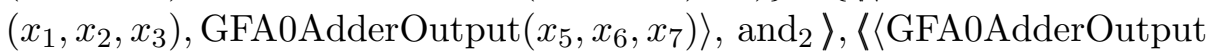
$\left.\left(x_{5}, x_{6}, x_{7}\right), x_{4}\right\rangle$, and $\left._{2}\right\rangle,\left\langle\left\langle x_{4}\right.\right.$, GFA0AdderOutput $\left.\left.\left(x_{1}, x_{2}, x_{3}\right)\right\rangle, \operatorname{and}_{2}\right\rangle$, GFA0 CarryOutput(GFA0AdderOutput $\left(x_{1}, x_{2}, x_{3}\right)$, GFA0AdderOutput $\left(x_{5}, x_{6}, x_{7}\right)$, 
$\left.\left.x_{4}\right)\right\} \cup\left\{\left\langle\left\langle\right.\right.\right.$ GFA0CarryOutput $\left(x_{1}, x_{2}, x_{3}\right)$, GFA0CarryOutput $\left.\left(x_{5}, x_{6}, x_{7}\right)\right\rangle$, xor $\left._{2}\right\rangle$, GFA0AdderOutput(GFA0CarryOutput $\left(x_{1}, x_{2}, x_{3}\right)$, GFA0Carry $\operatorname{Output}\left(x_{5}, x_{6}, x_{7}\right)$, GFA0CarryOutput(GFA0AdderOutput $\left(x_{1}, x_{2}, x_{3}\right)$, $\left.\left.\left.\operatorname{GFA0AdderOutput}\left(x_{5}, x_{6}, x_{7}\right), x_{4}\right)\right)\right\} \cup\left\{\left\langle\left\langle\operatorname{GFA0CarryOutput}\left(x_{1}, x_{2}, x_{3}\right)\right.\right.\right.$, GFA0CarryOutput $\left.\left(x_{5}, x_{6}, x_{7}\right)\right\rangle$, $\left.\operatorname{and}_{2}\right\rangle$, 〈〈GFA0CarryOutput $\left(x_{5}, x_{6}, x_{7}\right)$, GFA0CarryOutput(GFA0AdderOutput $\left(x_{1}, x_{2}, x_{3}\right)$, GFA0AdderOutput $\left.\left.\left(x_{5}, x_{6}, x_{7}\right), x_{4}\right)\right\rangle$, and 2$\rangle,\left\langle\left\langle\right.\right.$ GFA0CarryOutput(GFA0AdderOutput $\left(x_{1}, x_{2}\right.$, $\left.x_{3}\right)$, GFA0AdderOutput $\left.\left(x_{5}, x_{6}, x_{7}\right), x_{4}\right)$, GFA0CarryOutput $\left.\left(x_{1}, x_{2}, x_{3}\right)\right\rangle$, $\left.\operatorname{and}_{2}\right\rangle$, GFA0CarryOutput(GFA0CarryOutput $\left(x_{1}, x_{2}, x_{3}\right)$, GFA0Carry $\operatorname{Output}\left(x_{5}, x_{6}, x_{7}\right)$, GFA0CarryOutput(GFA0AdderOutput $\left(x_{1}, x_{2}, x_{3}\right)$, $\left.\left.\left.\operatorname{GFA0AdderOutput}\left(x_{5}, x_{6}, x_{7}\right), x_{4}\right)\right)\right\}$. The theorem is a consequence of $(21)$ and (10).

(23) InnerVertices( $\left.\operatorname{STC} 0 \operatorname{Str}\left(x_{1}, x_{2}, x_{3}, x_{4}, x_{5}, x_{6}, x_{7}\right)\right)$ is a binary relation.

Let us consider non pair sets $x_{1}, x_{2}, x_{3}, x_{4}, x_{5}, x_{6}, x_{7}$.

(24) InputVertices(STC0Str $\left.\left(x_{1}, x_{2}, x_{3}, x_{4}, x_{5}, x_{6}, x_{7}\right)\right)=\left\{x_{1}, x_{2}, x_{3}, x_{4}, x_{5}, x_{6}\right.$, $\left.x_{7}\right\}$. The theorem is a consequence of (10), (14), and (13).

(25) InputVertices(STC0Str $\left.\left(x_{1}, x_{2}, x_{3}, x_{4}, x_{5}, x_{6}, x_{7}\right)\right)$ has no pairs. The theorem is a consequence of (24).

Let us consider sets $x_{1}, x_{2}, x_{3}, x_{4}, x_{5}, x_{6}, x_{7}$.

(26) $x_{1}, x_{2}, x_{3}, x_{4}, x_{5}, x_{6}, x_{7},\left\langle\left\langle x_{1}, x_{2}\right\rangle\right.$, xor $\left._{2}\right\rangle,\left\langle\left\langle x_{1}, x_{2}\right\rangle, \operatorname{and}_{2}\right\rangle,\left\langle\left\langle x_{2}, x_{3}\right\rangle\right.$, $\left.\operatorname{and}_{2}\right\rangle,\left\langle\left\langle x_{3}, x_{1}\right\rangle, \operatorname{and}_{2}\right\rangle,\left\langle\left\langle x_{5}, x_{6}\right\rangle\right.$, xor $\left._{2}\right\rangle,\left\langle\left\langle x_{5}, x_{6}\right\rangle, \operatorname{and}_{2}\right\rangle,\left\langle\left\langle x_{6}, x_{7}\right\rangle, \operatorname{and}_{2}\right\rangle$, $\left\langle\left\langle x_{7}, x_{5}\right\rangle, \operatorname{and}_{2}\right\rangle$, GFA0AdderOutput $\left(x_{1}, x_{2}, x_{3}\right)$, GFA0CarryOutput $\left(x_{1}, x_{2}\right.$, $\left.x_{3}\right)$, GFA0AdderOutput $\left(x_{5}, x_{6}, x_{7}\right)$, GFA0CarryOutput $\left(x_{5}, x_{6}, x_{7}\right)$, 〈 GFA0 AdderOutput $\left(x_{1}, x_{2}, x_{3}\right)$, GFA0AdderOutput $\left.\left(x_{5}, x_{6}, x_{7}\right)\right\rangle$, xor $\left._{2}\right\rangle$, $\langle\langle$ GFA0Ad

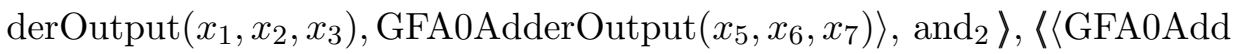
$\left.\left.\operatorname{erOutput}\left(x_{5}, x_{6}, x_{7}\right), x_{4}\right\rangle, \operatorname{and}_{2}\right\rangle,\left\langle\left\langle x_{4}, \operatorname{GFA0AdderOutput}\left(x_{1}, x_{2}, x_{3}\right)\right\rangle\right.$, and $\left.{ }_{2}\right\rangle$ $\in$ the carrier of $\operatorname{STC} 0 \operatorname{Str}\left(x_{1}, x_{2}, x_{3}, x_{4}, x_{5}, x_{6}, x_{7}\right)$.

And also GFA0AdderOutput(GFA0AdderOutput $\left(x_{1}, x_{2}, x_{3}\right)$, GFA0Add erOutput $\left.\left(x_{5}, x_{6}, x_{7}\right), x_{4}\right)$, GFA0CarryOutput(GFA0AdderOutput $\left(x_{1}, x_{2}, x_{3}\right)$, GFA0AdderOutput $\left.\left(x_{5}, x_{6}, x_{7}\right), x_{4}\right)$, 〈 $\left\langle\right.$ GFA0CarryOutput $\left(x_{1}, x_{2}, x_{3}\right)$, GFA0 CarryOutput $\left.\left(x_{5}, x_{6}, x_{7}\right)\right\rangle$, $\left.\operatorname{xor}_{2}\right\rangle$, GFA0AdderOutput(GFA0CarryOutput $\left(x_{1}\right.$, $\left.x_{2}, x_{3}\right)$, GFA0CarryOutput $\left(x_{5}, x_{6}, x_{7}\right)$, GFA0CarryOutput(GFA0AdderOut$\operatorname{put}\left(x_{1}, x_{2}, x_{3}\right)$, GFA0AdderOuput $\left.\left.\left(x_{5}, x_{6}, x_{7}\right), x_{4}\right)\right)$, 〈 GFA0CarryOutput $\left(x_{1}\right.$, $\left.x_{2}, x_{3}\right)$, GFA0CarryOutput $\left.\left(x_{5}, x_{6}, x_{7}\right)\right\rangle$, and $\left.{ }_{2}\right\rangle,\left\langle\left\langle\right.\right.$ GFA0CarryOutput $\left(x_{5}, x_{6}\right.$, $\left.x_{7}\right)$, GFA0CarryOutput(GFA0AdderOutput $\left(x_{1}, x_{2}, x_{3}\right)$, GFA0AdderOutput $\left.\left.\left(x_{5}, x_{6}, x_{7}\right), x_{4}\right)\right\rangle$, and $\left.{ }_{2}\right\rangle,\left\langle\left\langle\right.\right.$ GFA0CarryOutput(GFA0AdderOutput $\left(x_{1}, x_{2}, x_{3}\right)$, GFA0AdderOutput $\left.\left(x_{5}, x_{6}, x_{7}\right), x_{4}\right)$, GFA0CarryOutput $\left.\left.\left(x_{1}, x_{2}, x_{3}\right)\right\rangle, \operatorname{and}_{2}\right\rangle$, GFA0CarryOutput(GFA0CarryOutput $\left(x_{1}, x_{2}, x_{3}\right)$, GFA0CarryOutput $\left(x_{5}\right.$, $\left.x_{6}, x_{7}\right)$, GFA0CarryOutput(GFA0AdderOutput $\left(x_{1}, x_{2}, x_{3}\right)$, GFA0AdderOut 
$\left.\left.\operatorname{put}\left(x_{5}, x_{6}, x_{7}\right), x_{4}\right)\right) \in$ the carrier of $\operatorname{STC} 0 \operatorname{Str}\left(x_{1}, x_{2}, x_{3}, x_{4}, x_{5}, x_{6}, x_{7}\right)$. The theorem is a consequence of (15).

(27) $\left\langle\left\langle x_{1}, x_{2}\right\rangle\right.$, xor $\left._{2}\right\rangle,\left\langle\left\langle x_{1}, x_{2}\right\rangle\right.$, and $\left._{2}\right\rangle,\left\langle\left\langle x_{2}, x_{3}\right\rangle\right.$, and $\left._{2}\right\rangle,\left\langle\left\langle x_{3}, x_{1}\right\rangle, \operatorname{and}_{2}\right\rangle,\left\langle\left\langle x_{5}\right.\right.$, $\left.x_{6}\right\rangle$, xor $\left._{2}\right\rangle,\left\langle\left\langle x_{5}, x_{6}\right\rangle, \operatorname{and}_{2}\right\rangle,\left\langle\left\langle x_{6}, x_{7}\right\rangle, \operatorname{and}_{2}\right\rangle,\left\langle\left\langle x_{7}, x_{5}\right\rangle, \operatorname{and}_{2}\right\rangle$, GFA0Add erOutput $\left(x_{1}, x_{2}, x_{3}\right)$, GFA0CarryOutput $\left(x_{1}, x_{2}, x_{3}\right)$, GFA0AdderOutput $\left(x_{5}, x_{6}, x_{7}\right)$, GFA0CarryOutput $\left(x_{5}, x_{6}, x_{7}\right)$, 《/GFA0AdderOutput $\left(x_{1}, x_{2}\right.$, $\left.x_{3}\right)$,GFA0AdderOutput $\left.\left(x_{5}, x_{6}, x_{7}\right)\right\rangle$, ,or $\left._{2}\right\rangle,\left\langle\left\langle\operatorname{GFA0AdderOutput}\left(x_{1}, x_{2}, x_{3}\right)\right.\right.$, GFA0AdderOutput $\left.\left.\left(x_{5}, x_{6}, x_{7}\right)\right\rangle, \operatorname{and}_{2}\right\rangle,\left\langle\left\langle\operatorname{GFA0AdderOutput}\left(x_{5}, x_{6}, x_{7}\right)\right.\right.$, $\left.x_{4}\right\rangle$, and $\left._{2}\right\rangle,\left\langle\left\langle x_{4}\right.\right.$, GFA0AdderOutput $\left.\left(x_{1}, x_{2}, x_{3}\right)\right\rangle$, and $\left.{ }_{2}\right\rangle$, GFA0AdderOut put(GFA0AdderOutput $\left(x_{1}, x_{2}, x_{3}\right)$, GFA0AdderOutput $\left.\left(x_{5}, x_{6}, x_{7}\right), x_{4}\right)$ GFA0CarryOutput(GFA0AdderOutput $\left(x_{1}, x_{2}, x_{3}\right)$, GFA0AdderOutput $\left(x_{5}\right.$, $\left.\left.x_{6}, x_{7}\right), x_{4}\right)$, 〈(GFA0CarryOutput $\left.\left(x_{1}, x_{2}, x_{3}\right), \operatorname{GFA0CarryOutput}\left(x_{5}, x_{6}, x_{7}\right)\right\rangle$, $\left.\operatorname{xor}_{2}\right\rangle$, GFA0AdderOutput(GFA0CarryOutput $\left(x_{1}, x_{2}, x_{3}\right)$, GFA0CarryOut $\operatorname{put}\left(x_{5}, x_{6}, x_{7}\right)$, GFA0CarryOutput(GFA0AdderOutput $\left(x_{1}, x_{2}, x_{3}\right)$, GFA0 Adder Output $\left.\left.\left(x_{5}, x_{6}, x_{7}\right), x_{4}\right)\right) \in \operatorname{InnerVertices}\left(\operatorname{STC} 0 \operatorname{Str}\left(x_{1}, x_{2}, x_{3}, x_{4}, x_{5}\right.\right.$, $\left.x_{6}, x_{7}\right)$ ).

And also 〈 $/$ GFA0CarryOutput $\left(x_{1}, x_{2}, x_{3}\right)$, GFA0CarryOutput

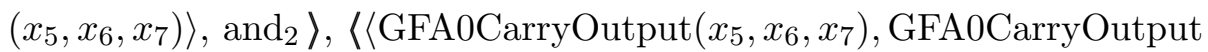
(GFA0AdderOutput $\left(x_{1}, x_{2}, x_{3}\right)$, GFA0AdderOutput $\left.\left.\left.\left(x_{5}, x_{6}, x_{7}\right), x_{4}\right)\right\rangle, \operatorname{and}_{2}\right\rangle$, 《/GFA0CarryOutput(GFA0AdderOutput $\left(x_{1}, x_{2}, x_{3}\right)$, GFA0AdderOutput $\left.\left(x_{5}, x_{6}, x_{7}\right), x_{4}\right)$, GFA0CarryOutput $\left.\left(x_{1}, x_{2}, x_{3}\right)\right\rangle$, and $\left.{ }_{2}\right\rangle$, GFA0CarryOutput (GFA0CarryOutput $\left(x_{1}, x_{2}, x_{3}\right)$, GFA0CarryOutput $\left(x_{5}, x_{6}, x_{7}\right)$, GFA0Carry Output(GFA0AdderOutput $\left(x_{1}, x_{2}, x_{3}\right)$, GFA0AdderOutput $\left.\left(x_{5}, x_{6}, x_{7}\right), x_{4}\right)$ ) $\in \operatorname{Inner} \operatorname{Vertices}\left(\operatorname{STC} 0 \operatorname{Str}\left(x_{1}, x_{2}, x_{3}, x_{4}, x_{5}, x_{6}, x_{7}\right)\right)$. The theorem is a consequence of $(22)$.

(28) Let us consider non pair sets $x_{1}, x_{2}, x_{3}, x_{4}, x_{5}, x_{6}, x_{7}$. Then $x_{1}, x_{2}$, $x_{3}, x_{4}, x_{5}, x_{6}, x_{7} \in \operatorname{InputVertices}\left(\operatorname{STC} 0 \operatorname{Str}\left(x_{1}, x_{2}, x_{3}, x_{4}, x_{5}, x_{6}, x_{7}\right)\right)$. The theorem is a consequence of (24).

Let $x_{1}, x_{2}, x_{3}, x_{4}, x_{5}, x_{6}, x_{7}$ be sets. The functors: $\operatorname{STC0OutS0}\left(x_{1}, x_{2}, x_{3}, x_{4}\right.$, $\left.x_{5}, x_{6}, x_{7}\right)$, STC0OutS1 $\left(x_{1}, x_{2}, x_{3}, x_{4}, x_{5}, x_{6}, x_{7}\right)$, and STC0OutS2 $\left(x_{1}, x_{2}, x_{3}\right.$, $\left.x_{4}, x_{5}, x_{6}, x_{7}\right)$ yielding elements of InnerVertices(STC0Str $\left(x_{1}, x_{2}, x_{3}, x_{4}, x_{5}, x_{6}\right.$, $\left.x_{7}\right)$ ) are defined by terms

(Def. 15) GFA0AdderOutput(GFA0AdderOutput $\left(x_{1}, x_{2}, x_{3}\right)$, GFA0AdderOutput $\left.\left(x_{5}, x_{6}, x_{7}\right), x_{4}\right)$,

(Def. 16) GFA0AdderOutput(GFA0CarryOutput $\left(x_{1}, x_{2}, x_{3}\right)$, GFA0CarryOutput $\left(x_{5}, x_{6}, x_{7}\right)$, GFA0CarryOutput(GFA0AdderOutput $\left(x_{1}, x_{2}, x_{3}\right)$, GFA0Ad$\left.\left.\operatorname{derOutput}\left(x_{5}, x_{6}, x_{7}\right), x_{4}\right)\right)$,

(Def. 17) GFA0CarryOutput(GFA0CarryOutput $\left(x_{1}, x_{2}, x_{3}\right)$, GFA0CarryOutput $\left(x_{5}, x_{6}, x_{7}\right)$, GFA0CarryOutput(GFA0AdderOutput $\left(x_{1}, x_{2}, x_{3}\right)$, GFA0Ad- 
$\left.\left.\operatorname{derOutput}\left(x_{5}, x_{6}, x_{7}\right), x_{4}\right)\right)$,

respectively. Now we state the propositions:

(29) Let us consider non pair sets $x_{1}, x_{2}, x_{3}, x_{4}, x_{5}, x_{6}, x_{7}$, a state $s$ of $\operatorname{STC} 0 \operatorname{Circ}\left(x_{1}, x_{2}, x_{3}, x_{4}, x_{5}, x_{6}, x_{7}\right)$, and elements $a_{1}, a_{2}, a_{3}, a_{4}, a_{5}, a_{6}, a_{7}$ of Boolean. Suppose $a_{1}=s\left(x_{1}\right)$ and $a_{2}=s\left(x_{2}\right)$ and $a_{3}=s\left(x_{3}\right)$ and $a_{4}=s\left(x_{4}\right)$ and $a_{5}=s\left(x_{5}\right)$ and $a_{6}=s\left(x_{6}\right)$ and $a_{7}=s\left(x_{7}\right)$. Then

(i) (Following $(s, 4))\left(\operatorname{STC} 0 O u t S 0\left(x_{1}, x_{2}, x_{3}, x_{4}, x_{5}, x_{6}, x_{7}\right)\right)=$ $\left.\left(\left(\left(\left(a_{1} \oplus a_{2}\right) \oplus a_{3}\right) \oplus a_{4}\right) \oplus a_{5}\right) \oplus a_{6}\right) \oplus a_{7}$, and

(ii) $($ Following $(s, 6))\left(\operatorname{STC0OutS1}\left(x_{1}, x_{2}, x_{3}, x_{4}, x_{5}, x_{6}, x_{7}\right)\right)=\left(\left(\left(a_{1} \wedge a_{2} \vee\right.\right.\right.$ $\left.\left.\left.a_{2} \wedge a_{3}\right) \vee a_{3} \wedge a_{1}\right) \oplus\left(\left(a_{5} \wedge a_{6} \vee a_{6} \wedge a_{7}\right) \vee a_{7} \wedge a_{5}\right)\right) \oplus\left(\left(\left(\left(a_{1} \oplus a_{2}\right) \oplus\right.\right.\right.$ $\left.\left.\left.a_{3}\right) \wedge\left(\left(a_{5} \oplus a_{6}\right) \oplus a_{7}\right) \vee\left(\left(a_{5} \oplus a_{6}\right) \oplus a_{7}\right) \wedge a_{4}\right) \vee a_{4} \wedge\left(\left(a_{1} \oplus a_{2}\right) \oplus a_{3}\right)\right)$, and

(iii) (Following $(s, 6))\left(\operatorname{STC} 0\right.$ OutS2 $\left.\left(x_{1}, x_{2}, x_{3}, x_{4}, x_{5}, x_{6}, x_{7}\right)\right)=\left(\left(\left(a_{1} \wedge a_{2} \vee\right.\right.\right.$ $\left.\left.a_{2} \wedge a_{3}\right) \vee a_{3} \wedge a_{1}\right) \wedge\left(\left(a_{5} \wedge a_{6} \vee a_{6} \wedge a_{7}\right) \vee a_{7} \wedge a_{5}\right) \vee\left(\left(a_{5} \wedge a_{6} \vee a_{6} \wedge a_{7}\right) \vee\right.$ $\left.a_{7} \wedge a_{5}\right) \wedge\left(\left(\left(\left(a_{1} \oplus a_{2}\right) \oplus a_{3}\right) \wedge\left(\left(a_{5} \oplus a_{6}\right) \oplus a_{7}\right) \vee\left(\left(a_{5} \oplus a_{6}\right) \oplus a_{7}\right) \wedge a_{4}\right) \vee\right.$ $\left.\left.a_{4} \wedge\left(\left(a_{1} \oplus a_{2}\right) \oplus a_{3}\right)\right)\right) \vee\left(\left(\left(\left(a_{1} \oplus a_{2}\right) \oplus a_{3}\right) \wedge\left(\left(a_{5} \oplus a_{6}\right) \oplus a_{7}\right) \vee\left(\left(a_{5} \oplus\right.\right.\right.\right.$ $\left.\left.\left.\left.a_{6}\right) \oplus a_{7}\right) \wedge a_{4}\right) \vee a_{4} \wedge\left(\left(a_{1} \oplus a_{2}\right) \oplus a_{3}\right)\right) \wedge\left(\left(a_{1} \wedge a_{2} \vee a_{2} \wedge a_{3}\right) \vee a_{3} \wedge a_{1}\right)$, and

(iv) (Following $(s, 6))\left(x_{1}\right)=a_{1}$, and

(v) (Following $(s, 6))\left(x_{2}\right)=a_{2}$, and

(vi) (Following $(s, 6))\left(x_{3}\right)=a_{3}$, and

(vii) (Following $(s, 6))\left(x_{4}\right)=a_{4}$, and

(viii) (Following $(s, 6))\left(x_{5}\right)=a_{5}$, and

(ix) (Following $(s, 6))\left(x_{6}\right)=a_{6}$, and

(x) $($ Following $(s, 6))\left(x_{7}\right)=a_{7}$.

(30) Let us consider non pair sets $x_{1}, x_{2}, x_{3}, x_{4}, x_{5}, x_{6}, x_{7}$, and a state $s$ of $\operatorname{STC} 0 \operatorname{Circ}\left(x_{1}, x_{2}, x_{3}, x_{4}, x_{5}, x_{6}, x_{7}\right)$. Then Following $(s, 6)$ is stable. The theorem is a consequence of (20).

\section{REFERENCES}

[1] Grzegorz Bancerek and Yatsuka Nakamura. Full adder circuit. Part I. Formalized Mathematics, 5(3):367-380, 1996.

[2] Grzegorz Bancerek, Czesław Byliński, Adam Grabowski, Artur Korniłowicz, Roman Matuszewski, Adam Naumowicz, Karol Pąk, and Josef Urban. Mizar: State-of-the-art and beyond In Manfred Kerber, Jacques Carette, Cezary Kaliszyk, Florian Rabe, and Volker Sorge, editors, Intelligent Computer Mathematics, volume 9150 of Lecture Notes in Computer Science, pages 261-279. Springer International Publishing, 2015. ISBN 978-3319-20614-1. doi $10.1007 / 978-3-319-20615-8 \_17$. 
[3] Grzegorz Bancerek, Czesław Byliński, Adam Grabowski, Artur Korniłowicz, Roman Matuszewski, Adam Naumowicz, and Karol Pak. The role of the Mizar Mathematical Library for interactive proof development in Mizar. Journal of Automated Reasoning, 61(1):9-32, 2018. do1 $10.1007 /$ s10817-017-9440-6

[4] Adam Grabowski, Artur Korniłowicz, and Adam Naumowicz. Four decades of Mizar. Journal of Automated Reasoning, 55(3):191-198, 2015. doi 10.1007/s10817-015-9345-1.

[5] Naoki Iwasaki and Katsumi Wasaki. A meta hardware description language melasy for model-checking systems. In Proceedings 5th International Conference on Information Technoloqy: New Generations (ITNG 2008), pages 273-278, 2008. doi:10.1109/ITNG.2008.135.

[6] Mayur Mehta, Vijay Parmar, and Earl Swartzlander. High-speed multiplier design using multi-input counter and compressor circuits. In Proceedinqs 10th IEEE Symposium on Computer Arithmetic, pages 43-50, June 1991. doi 10.1109/ARITH.1991.145532.

[7] Yatsuka Nakamura and Grzegorz Bancerek. Combining of circuits. Formalized Mathematics, 5(2):283-295, 1996.

[8] Yatsuka Nakamura, Piotr Rudnicki, Andrzej Trybulec, and Pauline N. Kawamoto. Introduction to circuits, I Formalized Mathematics, 5(2):227-232, 1996.

[9] Yatsuka Nakamura, Piotr Rudnicki, Andrzej Trybulec, and Pauline N. Kawamoto. Introduction to circuits, II. Formalized Mathematics, 5(2):273-278, 1996.

[10] Jean Vuillemin. A very fast multiplication algorithm for VLSI implementation. Integration, 1(1):39-52, 1983. doi $10.1016 / 0167-9260(83) 90005-6$.

[11] Christopher Stewart Wallace. A suggestion for a fast multiplier. IEEE Transactions on Electronic Computers, EC-13(1):14-17, 1964. doi 10.1109/PGEC.1964.263830.

[12] Katsumi Wasaki. Stability of the 4-2 binary addition circuit cells. Part I. Formalized Mathematics, 16(4):377-387, 2008. doi 10.2478/v10037-008-0046-7.

[13] Katsumi Wasaki and Pauline N. Kawamoto. 2's complement circuit. Formalized Mathematics, 6(2):189-197, 1997.

[14] Shin'nosuke Yamaguchi, Katsumi Wasaki, and Nobuhiro Shimoi. Generalized full adder circuits (GFAs). Part I. Formalized Mathematics, 13(4):549-571, 2005.

Accepted December 30, 2019 